Proceedings of the Edinburgh Mathematical Society (2006) 49, 657-666 (C)

DOI:10.1017/S001309150500057X Printed in the United Kingdom

\title{
APPROXIMATING NUMBERS WITH MISSING DIGITS BY ALGEBRAIC NUMBERS
}

\author{
SIMON KRISTENSEN* \\ School of Mathematics, University of Edinburgh, James Clerk Maxwell Building, \\ King's Buildings, Mayfield Road, Edinburgh EH9 3JZ, UK
}

(Received 19 April 2005)

\begin{abstract}
We show that for a given base $b$ and a proper subset $E \subset\{0, \ldots, b-1\}$, \#E<b-1, the set of numbers $x \in[0,1]$ that have no digits from $E$ in their expansion to base $b$ consists almost exclusively of $S^{*}$-numbers of type at most $\min \{2, \log b / \log (b-\# E)\}$. We also give upper bounds on the Hausdorff dimension of some exceptional sets.
\end{abstract}

Keywords: Diophantine approximation; transcendental numbers; Koksma's classification; Cantor sets; Hausdorff dimension

2000 Mathematics subject classification: Primary 11J83; 11J82

\section{Introduction}

Let $K \subseteq[0,1]$ be a compact set and suppose that $K$ supports a measure $\mu$ such that, for constants $\delta \in(0,1]$ and $c_{1}, c_{2}>0$,

$$
c_{1} r^{\delta} \leqslant \mu([c-r ; c+r]) \leqslant c_{2} r^{\delta}
$$

for all $c \in K$ and $r>0$ small enough. It is easy to see that any non-atomic measure supported on $K$ satisfying hypothesis (1.1) must also satisfy

$$
\mu([c-\varepsilon r ; c+\varepsilon r]) \leqslant c_{3} \varepsilon^{\delta} \mu([c-r ; c+r])
$$

for some $c_{3}>0$, whenever $r$ and $\varepsilon$ are small and $c \in \mathbb{R}$. This is the appropriate onedimensional specialization of the notion of an absolutely $\delta$-decaying measure used in $[\mathbf{4}, \mathbf{9}]$. Note that in this case, the intervals considered are centred anywhere on the real axis. This will be used in the proof, and can be easily deduced from the less general statement when $c \in K$ (see the remarks following the definition of an absolutely $\delta$-decaying measure in $[9])$. Here and subsequently we will assume that $\mu$ has been normalized so that $\mu(K)=1$.

Properties (1.1) and (1.2) are important because of the following theorem, which combines specializations of a theorem by Hutchinson $[\mathbf{3}]$ and of Kleinbock, Lindenstrauss and Weiss [4] with [2, Theorem 9.3].

* Present address: Department of Mathematical Sciences, Faculty of Science, University of Aarhus, Ny Munkegade, Building 530, 8000 Aarhus C, Denmark (sik@imf.au.dk). 
Theorem. Let $\left\{h_{1}, \ldots, h_{t}\right\}$ be a family of affine contractions of $\mathbb{R}$, such that, for some open set $U \subseteq \mathbb{R}$,

$$
h_{i}(U) \subseteq U \quad \text { for all } i=1, \ldots t, \quad \text { and } \quad i \neq j \Rightarrow h_{i}(U) \cap h_{j}(U)=\emptyset .
$$

Suppose further that no finite set $\left\{x_{1}, \ldots, x_{M}\right\}$ is invariant under the full family $\left\{h_{1}, \ldots, h_{t}\right\}$. There is then a unique non-empty compact set $K$ such that

$$
K=\bigcup_{i=1}^{t} h_{i}(K),
$$

which supports a non-atomic measure satisfying condition (1.1) (and consequently condition (1.2)). Furthermore, $\delta$ is the positive real number satisfying

$$
\sum_{i=1}^{t} \rho_{i}^{\delta}=1,
$$

where for each $i \in\{1, \ldots, t\}, \rho_{i} \in(0,1)$ is the real number such that $h_{i}(x)= \pm \rho_{i} x+\tau_{i}$ for some $\tau_{i} \in \mathbb{R}$.

Condition (1.3) is known as the open set condition, and is sufficient to ensure the existence of the set $K$. The additional restriction that no finite set is invariant under the action of the family is to ensure that the limiting measure is non-atomic. The theorem has a higher-dimensional generalization, but this is not relevant for the purposes of this paper.

Let $b \in \mathbb{N}, b>2$, and let $E \subset\{0, \ldots, b-1\}$. Consider the set $C_{b, E} \subseteq[0,1]$ of numbers whose expansion to base $b$ does not contain any of the digits in $E$. This generalizes the well-known ternary Cantor set, which is obtained when $b=3$ and $E=\{1\}$. Of course, if $\# E=b-1$, the set $C_{b, E}$ consists of a single point and $\delta=0$. We will disregard this degenerate case and assume that $\# E<b-1$. It is straightforward to construct a family of contractions having $C_{b, E}$ as their limit set (see, for example, $[\mathbf{3}]$ ). One can easily show that this family satisfies the conditions of the above theorem. By that theorem, $C_{b, E}$ supports a measure satisfying (1.1). Consequently, in all results below, we may read $C_{b, E}$ for $K$ to obtain statements about these sets. Also, we easily see that $\delta=\log (b-\# E) / \log b$.

Let $n \in \mathbb{N}$ and let $\mathbb{A}_{n}$ denote the set of real algebraic numbers of degree less than or equal to $n$. For an algebraic number $\alpha$, we denote its height by $H(\alpha)$, i.e. the maximum modulus of the coefficients of the minimal polynomial of $\alpha$. We are concerned with the approximation of elements of $K$ by elements of $\mathbb{A}_{n}$, where the quality of approximation is measured in terms of the height of the approximating number. Let $\psi: \mathbb{R}_{\geqslant 1} \rightarrow \mathbb{R}_{>0}$. We define the set

$$
\mathcal{K}_{n}^{*}(\psi ; K)=\left\{x \in K:|x-\alpha|<\psi(H(\alpha)) \text { for infinitely many } \alpha \in \mathbb{A}_{n}\right\} .
$$

The set $\mathcal{K}_{n}^{*}(\psi ;[0,1])$ has been widely studied (see $\left.[\mathbf{1}]\right)$. When $n=1$, we are approximating elements of $K$ by rationals, and further results on the measure and dimension are known 
$[\mathbf{4}, \mathbf{5}, \mathbf{9}, \mathbf{1 0}]$. In this paper we are interested in finding upper bounds for the measure and Hausdorff dimension of the sets $\mathcal{K}_{n}^{*}(\psi ; K)$, where $K$ is subject to condition (1.1). Particular examples of such sets are the $C_{b, E}$, with the only restriction that $\# E<b-1$. This has some number-theoretic consequences.

We briefly mention some related questions and results. Mahler [8] asked how closely an element in the ternary Cantor set can be approximated by rationals (see also [7] and [6], where it is conjectured that the sets $C_{b, E}$ contain no algebraic irrationals). The partial answer by Weiss $[\mathbf{1 0}]$ was 'almost surely not better than expected'. The present paper gives a similar answer for approximation by algebraic numbers of bounded degree.

\section{Statement of results}

We first find a criterion on the function $\psi$ under which we are guaranteed that the set $\mathcal{K}_{n}^{*}(\psi ; K)$ is null with respect to $\mu$. We obtain the following theorem.

Theorem 2.1. Let $K \subseteq[0,1]$ be a compact set supporting a measure $\mu$ satisfying (1.2). Suppose that $\psi: \mathbb{R}_{\geqslant 1} \rightarrow \mathbb{R}_{\geqslant 0}$ satisfies either

$$
\sum_{r=1}^{\infty} r^{2 n \delta-1} \psi(r)^{\delta}<\infty \text { and } \psi \text { is non-increasing }
$$

or

$$
\sum_{r=1}^{\infty} r^{n} \psi(r)^{\delta}<\infty
$$

Then

$$
\mu\left(\mathcal{K}_{n}^{*}(\psi ; K)\right)=0
$$

When $n=1$ and $K=C_{3,\{1\}}$, this reduces to the theorem of [10]. Note that whenever $\delta>(n+1) / 2 n$, the first convergence condition is stronger than the second. Note also that the monotonicity assumption is only needed in the case when the first series is convergent.

In Koksma's classification of transcendental numbers, we encounter the quantities

$$
w_{n}^{*}(x)=\sup \left\{w>0:|x-\alpha|<H(\alpha)^{-w-1} \text { for infinitely many } \alpha \in \mathbb{A}_{n}\right\}
$$

and

$$
w^{*}(x)=\limsup _{n \rightarrow \infty} \frac{w_{n}^{*}(x)}{n},
$$

defined for any transcendental number $x$. If $w^{*}(x)<\infty$, then $x$ is said to be an $S^{*}$ number of type $w^{*}(x)$. Note that we are using the definitions from [1] (see the discussion in that book for alternative definitions of the quantities used). We have the following corollary to Theorem 2.1.

Corollary 2.2. For $\mu$-almost every $x \in K, w_{n}^{*}(x) \leqslant \min \{2 n-1,(n+1-\delta) / \delta\}$. Consequently, $\mu$-almost every $x \in K$ is an $S^{*}$-number of type at most $\min \{2,1 / \delta\}$. 
Note that we have lost some information by restricting to a Cantor set. Indeed, it is well known that almost all real numbers are $S^{*}$-numbers of type $=1$ (see, for example, $[\mathbf{1}$, Corollary 4.1]). At present, I do not know if the bound on the type can be improved for general sets satisfying (1.1).

In analogy with Koksma's classification, we have Mahler's classification (which actually predates Koksma's). In this classification, we have quantities

$$
w_{n}(x)=\sup \left\{w>0:|P(x)|<H(P)^{-w} \text { for infinitely many } P \in \mathbb{Z}[X], \operatorname{deg}(P) \leqslant n\right\}
$$

and

$$
w(x)=\limsup _{n \rightarrow \infty} \frac{w_{n}(x)}{n} .
$$

If $0<w(x)<\infty$, then $x$ is said to be an $S$-number of type $w(x)$. We now have a second corollary to Theorem 2.1 .

Corollary 2.3. For $\mu$-almost every $x \in K, w_{n}(x) \leqslant \min \{3 n-2,((1+\delta) n+1-2 \delta) / \delta\}$. Consequently, $\mu$-almost every $x \in K$ is an $S$-number of type at most $\min \{3,(1+\delta) / \delta\}$.

We now turn our attention to the Hausdorff dimension of the null sets arising from Theorem 2.1. Denote by $\mathcal{H}^{s}(E)$ the $s$-dimensional Hausdorff measure of the set $E$ and by $\operatorname{dim}_{\mathrm{H}}(E)$ the Hausdorff dimension of $E$ (see, for example, $[\mathbf{2}]$ for the definitions). If $K$ supports a measure satisfying $(1.1)$, it follows directly that $\operatorname{dim}_{\mathrm{H}}(K)=\delta$. We now have the following theorem.

Theorem 2.4. Let $K \subseteq[0,1]$ be a compact set supporting a non-atomic measure $\mu$ satisfying (1.1). Let $s \in[0, \delta]$ and let $\psi: \mathbb{R}_{\geqslant 1} \rightarrow \mathbb{R}_{>0}$ be such that either

$$
\sum_{r=1}^{\infty} r^{2 n \delta-1} \psi(r)^{s}<\infty \text { and } \psi \text { is non-increasing }
$$

or

$$
\sum_{r=1}^{\infty} r^{n} \psi(r)^{s}<\infty
$$

Then

$$
\mathcal{H}^{s}\left(\mathcal{K}_{n}^{*}(\psi ; K)\right)=0 .
$$

From Theorem 2.4, we may deduce an upper bound on the Hausdorff dimension of the sets $\mathcal{K}_{n}^{*}(\psi ; K)$. For a function $\psi: \mathbb{R}_{\geqslant 1} \rightarrow \mathbb{R}_{>0}$, we define the lower order at infinity of $1 / \psi$ to be

$$
\lambda_{\psi}=\liminf _{r \rightarrow \infty} \frac{-\log \psi(r)}{\log r} .
$$

Corollary 2.5. Let $\psi: \mathbb{R}_{\geqslant 1} \rightarrow \mathbb{R}_{>0}$ be non-increasing with $\lambda_{\psi} \geqslant \min \{2 n,(n+1) / \delta\}$. Then

$$
\operatorname{dim}_{\mathrm{H}}\left(\mathcal{K}_{n}^{*}(\psi ; K)\right) \leqslant \min \left\{\frac{2 n \delta}{\lambda_{\psi}}, \frac{n+1}{\lambda_{\psi}}\right\} .
$$


Of course, as $\mathcal{K}_{n}^{*}(\psi ; K) \subseteq \mathcal{K}_{n}^{*}(\psi ;[0,1])$ and $\operatorname{as} \operatorname{dim}_{\mathrm{H}}\left(\mathcal{K}_{n}^{*}(\psi ;[0,1])\right)=(n+1) / \lambda_{\psi}$ under the same assumptions as in Corollary 2.5 (see [1, Theorem 6.7]), we recognize the second upper bound as the one of this theorem. The first estimate is stronger only if $\delta \leqslant(n+1) / 2 n$. This is certainly satisfied for all $n$ if $\delta \leqslant \frac{1}{2}$. For higher $\delta$, new information is only gained for suitably small $n$.

The results obtained in the present paper are unlikely to be best possible. This is a consequence of the methods used in the proofs, and we will make conjectures on the best possible results in the final section. To prove stronger results of the type in this paper, information on the distribution of all algebraic numbers of bounded degree near $K$ is needed. For the very general $K$ studied here, we do not have sufficiently accurate information to obtain the conjectured results. Instead, we make do with weak distributional results which hold on all of $\mathbb{R}$, and use measure-theoretic arguments to deduce distributional results for algebraic numbers near $K$. The difficulty in improving the results of the present paper is that these weak distributional results on $\mathbb{R}$ are, in a sense, best possible, as small gaps do occur between real algebraic numbers of bounded degree.

\section{Proof of Theorem 2.1}

We first prove that the convergence of the first series ensures that the measure is zero. This is by far the most difficult part of the proof. We will use a consequence of $[\mathbf{1}$, Corollary A.2]. It is a consequence of this corollary that if $\alpha$ and $\beta$ are distinct real algebraic numbers of degree at most $n$, then

$$
|\alpha-\beta| \geqslant c_{4} H(\alpha)^{-n} H(\beta)^{-n},
$$

where the constant $c_{4}>0$ depends solely on $n$. If, for some $k \in \mathbb{N}, 2^{k} \leqslant H(\alpha)$, $H(\beta)<2^{k+1}$, this implies that $|\alpha-\beta|>\frac{1}{2} c_{4} 2^{-2 n(k+1)}$. Consequently, for distinct real algebraic numbers $\alpha_{i}$ with $2^{k} \leqslant H\left(\alpha_{i}\right)<2^{k+1}$, the intervals

$$
\left[\alpha_{i}-\frac{1}{4} c_{4} 2^{-2 n(k+1)} ; \alpha_{i}+\frac{1}{4} c_{4} 2^{-2 n(k+1)}\right]
$$

are disjoint.

Let $k \in \mathbb{N}$. We will show that as $k \rightarrow \infty$,

$$
\max _{2^{k} \leqslant r<2^{k+1}} \frac{\psi(r)}{4^{-1} c_{4} 2^{-2 n(k+1)}}=o(1) .
$$

Indeed, suppose to the contrary that there is a $c_{5}>0$ and a strictly increasing sequence $\left\{k_{i}\right\}_{i=1}^{\infty} \subseteq \mathbb{N}$ such that for any $i \in \mathbb{N}$

$$
\max _{2^{k_{i} \leqslant r<2^{k_{i}+1}}} \frac{\psi(r)}{4^{-1} c_{4} 2^{-2 n\left(k_{i}+1\right)}}>c_{5} .
$$

By the convergence assumption of the theorem together with Cauchy's condensation criterion,

$$
\sum_{k=1}^{\infty} 2^{2 n(k+1) \delta} \psi\left(2^{k}\right)^{\delta}=2^{2 n \delta} \sum_{k=1}^{\infty}\left(2^{2 k n} \psi\left(2^{k}\right)\right)^{\delta}<\infty .
$$


On the other hand, as $\psi$ is non-increasing,

$$
\begin{aligned}
\sum_{k=1}^{\infty}\left(2^{2 n(k+1)} \psi\left(2^{k}\right)\right)^{\delta} & \geqslant 4^{-\delta} c_{4}^{\delta} \sum_{i=1}^{\infty}\left(\max _{2^{k_{i}} \leqslant r<2^{k_{i}+1}} \frac{\psi(r)}{4^{-1} c_{4} 2^{-2 n\left(k_{i}+1\right)}}\right)^{\delta} \\
& >4^{-\delta} c_{4}^{\delta} c_{5}^{\delta} \sum_{i=1}^{\infty} 1=\infty,
\end{aligned}
$$

which is the desired contradiction.

Consider the sets

$$
E_{k}=\bigcup_{\substack{\alpha \in \mathbb{A}_{n} \\ 2^{k} \leqslant H(\alpha)<2^{k+1}}}[\alpha-\psi(H(\alpha)) ; \alpha+\psi(H(\alpha))] .
$$

Clearly, for $k$ large enough,

$$
\begin{aligned}
\mu\left(E_{k}\right) \leqslant & \sum_{\substack{\alpha \in \mathbb{A}_{n} \\
2^{k} \leqslant H(\alpha)<2^{k+1}}} \mu([\alpha-\psi(H(\alpha)) ; \alpha+\psi(H(\alpha))]) \\
& \leqslant c_{3} c_{4}^{\delta} 4^{-\delta} 2^{2 n(k+1) \delta} \psi\left(2^{k}\right)^{\delta} \sum_{\substack{\alpha \in \mathbb{A}_{n} \\
2^{k} \leqslant H(\alpha)<2^{k+1}}} \mu\left(\left[\alpha-\frac{1}{4} c_{4} 2^{-2 n(k+1)} ; \alpha+\frac{1}{4} c_{4} 2^{-2 n(k+1)}\right]\right),
\end{aligned}
$$

where we have used (1.2) and (3.2). The intervals in the final sum are disjoint. Hence, the sum of their measure is bounded from above by the measure of $K$, which is equal to 1 . We have shown that for $k \geqslant k_{0}$,

$$
\mu\left(E_{k}\right) \leqslant c_{3} c_{4}^{\delta} 4^{-\delta} 2^{2 n(k+1) \delta} \psi\left(2^{k}\right)^{\delta} .
$$

To complete the proof of this case, we note that $\mathcal{K}_{n}^{*}(\psi ; K)$ is the set of points falling in infinitely many of the $E_{k}$. But

$$
\sum_{k=k_{0}}^{\infty} \mu\left(E_{k}\right) \leqslant c_{3} c_{4}^{\delta} 4^{-\delta} \sum_{k=k_{0}}^{\infty} 2^{2 n(k+1) \delta} \psi\left(2^{k}\right)^{\delta}=c_{3} c_{4}^{\delta} 4^{-\delta} 2^{2 n \delta} \sum_{k=k_{0}}^{\infty} 2^{2 n k \delta} \psi\left(2^{k}\right)^{\delta} .
$$

Using Cauchy's condensation criterion and the convergence assumption of the theorem, the latter series converges. Hence, the Borel-Cantelli lemma implies the theorem.

To show that the convergence of the second series is sufficient to ensure zero measure, we note that

$$
\#\left\{\alpha \in \mathbb{A}_{n}: \alpha \in[0,1], H(\alpha)=H\right\} \leqslant n(n+1)(2 H+1)^{n} .
$$

By (1.1), for any such $\alpha$, we have $\mu([\alpha-\psi(H) ; \alpha+\psi(H)]) \leqslant c_{6} \psi(H)^{\delta}$ for some $c_{6}>0$. Elements of $\mathcal{K}_{n}^{*}(\psi ; K)$ fall in infinitely many of these intervals, and as

$$
\sum_{\substack { H=1 \\
\begin{subarray}{c}{\left.\alpha \in \mathbb{A}_{n} \\
\alpha 0,1\right] \\
H(\alpha)=H{ H = 1 \\
\begin{subarray} { c } { \alpha \in \mathbb { A } _ { n } \\
\alpha 0 , 1 ] \\
H ( \alpha ) = H } }\end{subarray}} \mu([\alpha-\psi(H) ; \alpha+\psi(H)]) \leqslant n(n+1) c_{6} \sum_{H=1}^{\infty}(2 H+1)^{n} \psi(H)^{\delta},
$$


which converges by assumption, the measure of $\mathcal{K}_{n}^{*}(\psi ; K)$ is zero by the Borel-Cantelli lemma.

Proof of Corollary 2.2. Let $n \in \mathbb{N}$ be fixed and let $x \in K$. Then, for any $m \in \mathbb{N}$, $x \in \mathcal{K}_{n}^{*}\left(r \mapsto r^{-w_{n}^{*}(x)-1+1 / m} ; K\right)$.

Let $m \in \mathbb{N}$ be fixed but arbitrary and consider the set

$$
\mathcal{E}_{n}(m)=\left\{x \in K: w_{n}^{*}(x)>\min \{2 n-1,(n+1-\delta) / \delta\}+2 / m\right\} .
$$

By the above argument,

$$
\mathcal{E}_{n}(m) \subseteq \mathcal{K}\left(r \mapsto r^{-2 n-1 / m} ; K\right) \cup \mathcal{K}\left(r \mapsto r^{-(n+1) / \delta-1 / m} ; K\right)
$$

But since

$$
\sum_{r=1}^{\infty} r^{2 n \delta-1} r^{(-2 n-1 / m) \delta}=\sum_{r=1}^{\infty} r^{-1-\delta / m}<\infty
$$

and

$$
\sum_{r=1}^{\infty} r^{n} r^{(-(n+1) / \delta-1 / m) \delta}=\sum_{r=1}^{\infty} r^{-1-\delta / m}<\infty
$$

the set on the left-hand side of (3.5) has measure zero by Theorem 2.1 , so $\mu\left(\mathcal{E}_{n}(m)\right)=0$.

Now consider the set

$$
\mathcal{E}=\bigcup_{n=1}^{\infty} \bigcup_{m=1}^{\infty} \mathcal{E}_{n}(m)
$$

Since $\mathcal{E}$ is a countable union of null sets, it is itself a null set. Consequently, almost every $x \in K$ is in the complement of $\mathcal{E}$, i.e. they satisfy, for any $n, m \in \mathbb{N}$,

$$
w_{n}^{*}(x) \leqslant \min \{2 n-1,(n+1-\delta) / \delta\}+2 / m,
$$

so that on letting $m$ tend to infinity, for any $n \in \mathbb{N}$,

$$
w_{n}^{*}(x) \leqslant \min \{2 n-1,(n+1-\delta) / \delta\},
$$

for $\mu$-almost every $x \in K$. This proves the first part of the corollary. To complete the proof, note that, for such $x$,

$$
w^{*}(x)=\limsup _{n \rightarrow \infty} \frac{w_{n}^{*}(x)}{n} \leqslant \limsup _{n \rightarrow \infty} \min \{(2 n-1) / n,(n+1-\delta) / n \delta\}=\min \{2,1 / \delta\} .
$$

Proof of Corollary 2.3. From [1, Theorem 3.4] we know that

$$
w_{n}(x) \leqslant w_{n}^{*}(x)+n-1 .
$$

Inserting the bounds of Corollary 2.2 into this inequality yields the first statement. The second statement is derived as in the proof of Corollary 2.2. Alternatively, it can be deduced directly from this corollary together with [1, Theorem 3.6]. 


\section{Proof of Theorem 2.4}

For each $k_{0} \in \mathbb{N}$, we will define a cover of $\mathcal{K}_{n}^{*}(\psi ; K)$ with intervals. Each of these will give an upper bound on the Hausdorff $s$-measure of $\mathcal{K}_{n}^{*}(\psi ; K)$, which will tend to zero as $k_{0}$ tends to infinity. This will imply the theorem.

Suppose initially that the first series converges. We define

$$
\mathcal{D}_{k}=\left\{\alpha \in \mathbb{A}_{n}: 2^{k} \leqslant H(\alpha)<2^{k+1},\left[\alpha-\psi\left(2^{k}\right) ; \alpha+\psi\left(2^{k}\right)\right] \cap K \neq \emptyset\right\} .
$$

With $c_{4}$ defined by (3.1), we see that by disjointness and by (1.1),

$$
\# \mathcal{D}_{k} c_{1} 4^{-\delta} c_{4}^{\delta} 2^{-2 n(k+1) \delta} \leqslant \mu\left(\bigcup_{\alpha \in \mathcal{D}_{k}}\left[\alpha-\frac{1}{4} c_{4} 2^{-2 n(k+1)} ; \alpha+\frac{1}{4} c_{4} 2^{-2 n(k+1)}\right]\right) \leqslant \mu(K)=1 .
$$

Consequently,

$$
\# \mathcal{D}_{k} \leqslant c_{1}^{-1} 4^{\delta} c_{4}^{-\delta} 2^{2 n(k+1) \delta} .
$$

For any $k_{0} \in \mathbb{N}$, the family

$$
\bigcup_{k=k_{0}}^{\infty} \bigcup_{\alpha \in \mathcal{D}_{k}}\left[\alpha-\psi\left(2^{k}\right) ; \alpha+\psi\left(2^{k}\right)\right]
$$

covers $\mathcal{K}_{n}^{*}(\psi ; K)$. Hence, for $s \in[0, \delta]$,

$$
\mathcal{H}^{s}\left(\mathcal{K}_{n}^{*}(\psi ; K)\right) \leqslant \sum_{k=k_{0}}^{\infty} \sum_{\alpha \in \mathcal{D}_{k}} 2^{s} \psi\left(2^{k}\right)^{s} \leqslant 2^{s} c_{1}^{-1} 4^{\delta} c_{4}^{-\delta} 2^{2 n \delta} \sum_{k=k_{0}}^{\infty} 2^{2 k n \delta} \psi\left(2^{k}\right)^{s} .
$$

Using Cauchy's condensation criterion, we see that the latter tends to zero as $k_{0}$ tends to infinity, by assumption.

Suppose now that the second series converges. In this case, the family

$$
\bigcup_{H=H_{0}}^{\infty} \bigcup_{\substack{\alpha \in \mathbb{A}_{n} \\ \alpha \in[0,1] \\ H(\alpha)=H}}[\alpha-\psi(H) ; \alpha+\psi(H)]
$$

covers $\mathcal{K}_{n}^{*}(\psi ; K)$ for any $H_{0} \in \mathbb{N}$. Using $(3.3)$,

$$
\mathcal{H}^{s}\left(\mathcal{K}_{n}^{*}(\psi ; K)\right) \leqslant \sum_{H=H_{0}}^{\infty} \sum_{\substack{\alpha \in \mathbb{A}_{n} \\ \alpha \in[0,1] \\ H(\alpha)=H}} 2^{s} \psi(H)^{s} \leqslant 2^{s} n(n+1) \sum_{H=H_{0}}^{\infty}(2 H+1)^{n} \psi(H)^{s},
$$

which tends to zero as $H_{0}$ tends to infinity, by assumption.

Proof of Corollary 2.5. Let $\eta>0$ be fixed and let $s=2 n \delta / \lambda_{\psi}+\eta$. Choose $\varepsilon<$ $2 n \delta \eta\left(2 n \delta / \lambda_{\psi}+\eta\right)^{-1}$ and let $r_{0}$ be sufficiently large that for $r \geqslant r_{0}, \log \psi(r) / \log r \leqslant$ $-\lambda_{\psi}+\varepsilon$. Then

$$
\sum_{r=r_{0}}^{\infty} r^{2 n \delta-1} \psi(r)^{s} \leqslant \sum_{r=r_{0}}^{\infty} r^{2 n \delta-1-2 n \delta-2 n \delta \eta+\varepsilon\left(2 n \delta / \lambda_{\psi}+\eta\right)}=\sum_{r=r_{0}}^{\infty} r^{-1-\varepsilon^{\prime}},
$$


where $\varepsilon^{\prime}=2 n \delta \eta-\varepsilon\left(2 n \delta / \lambda_{\psi}+\eta\right)>0$. Hence, the series converges and

$$
\operatorname{dim}_{\mathrm{H}}\left(\mathcal{K}_{n}^{*}(\psi ; K)\right) \leqslant \frac{2 n \delta}{\lambda_{\psi}}+\eta .
$$

As $\eta>0$ was arbitrary, the first upper bound of the corollary follows. The second upper bound follows as $\mathcal{K}_{n}^{*}(\psi ; K) \subseteq \mathcal{K}_{n}^{*}(\psi ;[0,1])$, so that

$$
\operatorname{dim}_{\mathrm{H}}\left(\mathcal{K}_{n}^{*}(\psi ; K)\right) \leqslant \operatorname{dim}_{\mathrm{H}}\left(\mathcal{K}_{n}^{*}(\psi ;[0,1])\right)=(n+1) / \lambda_{\psi}
$$

by $[\mathbf{1}$, Theorem 6.7$]$. Of course, this could also be shown to follow from the convergence of the second series of Theorem 2.4 .

\section{Concluding remarks}

The results of this paper are unlikely to be the best possible, except possibly when $n=1$ where approximation by rationals is considered. The reason for this is the use of inequality (3.1). When $n=1$, this is sharp, since for $p / q, p^{\prime} / q^{\prime} \in \mathbb{Q}$ with $2^{k} \leqslant q$, $q^{\prime}<2^{k+1},\left|p / q-p^{\prime} / q^{\prime}\right| \geqslant 1 /\left(q q^{\prime}\right)>2^{-2(k+1)}$. Under the assumption, this is the best possible, since, if $\left(q, q^{\prime}\right)=1$, we may choose $p, p^{\prime}$ such that $q p^{\prime}-q^{\prime} p=1$. However, while gaps between real algebraic numbers as small as those postulated in inequality (3.1) do occur, it is not in general best possible, as such numbers are not as regularly distributed as rationals. Indeed, if it was best possible, we would have of the order of $2^{2 k n}$ algebraic numbers of degree less than or equal to $n$ and height $H \in\left[2^{k} ; 2^{k+1}\right)$ in the unit interval, but by (3.3), there are only of the order of $2^{k(n+1)}$ such numbers. Hence, there must be larger gaps between at least some of these numbers. Identifying these gaps is a difficult problem, and at present we have no way of ensuring that the large gaps do not all fall far away from a given set $K$. Hence, our result is not as strong as could be desired.

In the light of [1, Theorem 6.7], the sharpest upper bound is likely to be obtained when the exponent $2 n \delta-1$ of $r$ in the first series of Theorems 2.1 and 2.4 is replaced by $(n+1) \delta-1$. If such an upper bound could be shown to hold, it would imply that, given a set $K \subseteq[0,1]$ supporting a measure $\mu$ satisfying condition (1.1), $\mu$-almost all numbers in $K$ would be $S^{*}$-numbers of type at most 1 . It would also remove the restrictions on $\delta$ under which Corollary 2.5 improves upon what is known from [1, Theorem 6.7]. Better knowledge of the distribution of algebraic numbers than is used here is clearly needed in order to prove this. It will be the subject of further study.

Acknowledgements. I thank Yann Bugeaud for valuable discussions and for his comments on the various drafts of this paper. I also thank the Department of Mathematical Sciences at the University of Aarhus for their kind hospitality. Finally, I thank an anonymous referee for helpful suggestions.

The author is a William Gordon Seggie Brown Fellow.

\section{References}

1. Y. Bugeaud, Approximation by algebraic numbers, Cambridge Tracts in Mathematics, Volume 160 (Cambridge University Press, 2004). 
2. K. FALCONER, Fractal geometry-mathematical foundations and applications (Wiley, 1990).

3. J. E. Hutchinson, Fractals and self-similarity, Indiana Univ. Math. J. 30 (1981), 713747.

4. D. Kleinbock, E. Lindenstrauss and B. Weiss, On fractal measures and diophantine approximation, Selecta Math. 10(4) (2004), 479-523.

5. J. Levesley, C. Salp and S. Velani, On a problem of K. Mahler: Diophantine approximation and Cantor sets, arXiv eprint math.NT/0505074.

6. K. MAHLER, Lectures on transcendental numbers, Proceedings of Symposia in Pure Mathematics, Vol XX, pp. 248-274 (American Mathematical Society, Providence, RI, 1971).

7. K. MAHLER, Fifty years as a mathematician, J. Number Theory 14(2) (1982), 121-155.

8. K. MAhler, Some suggestions for further research, Bull. Austral. Math. Soc. 29(1) (1984), 101-108.

9. A. Pollington And S. Velani, Metric Diophantine approximation and 'absolutely friendly' measures, Selecta Math. 11(2) (2005), 297-307.

10. B. Weiss, Almost no points on a Cantor set are very well approximable, Proc. R. Soc. Lond. A 457 (2001), 949-952. 